\title{
EMPRESAS ESPAÑOLAS DE GESTIÓN DOCUMENTAL: SERVICIOS, ORGANIZACIÓN, EMPLEO Y PERSPECTIVAS
}

\author{
Antonio Paños-Álvarez y Pedro-José Saura-Meroño
}
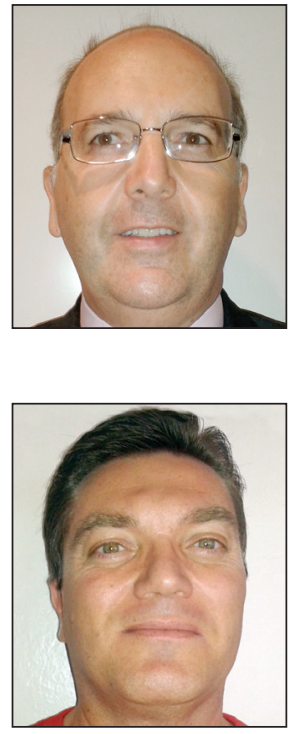

\section{Resumen}

Los estudios sobre la clasificación y evolución de las actividades de negocio de las empresas españolas de servicios documentales y sus características organizativas, políticas de empleo y otras cuestiones han sido escasos en los últimos años. Conscientes de la importancia que estos temas tienen para los centros universitarios y los estudiantes de las titulaciones de información y documentación, se presentan los resultados obtenidos del análisis de estas empresas y su comparación con los datos disponibles de estudios anteriores. Se presenta los tipos de servicios documentales que ofrecen estas empresas, su distribución geográfica, ámbito de negocio, tamaño y edad, datos actuales sobre sus políticas de empleo y selección y perspectivas de futuro ante la actual situación de crisis. Aportaciones novedosas de este estudio son el análisis del ámbito de negocio y la edad de estas empresas, así como los factores que tienen en cuenta en la selección de empleados.

\section{Palabras clave}

Empresas, Servicios documentales, España, Edad, Tamaño, Localización, Empleo, Selección de personal, Expectativas de futuro.

\section{Title: Spanish companies offering information management: services, organization, employment and} prospects

\begin{abstract}
In recent years, very few studies have classified and analysed the evolution of the business activities carried out by Spanish companies that provide information and document management services. Aware of the importance that these issues have for university centers and for graduate students of library and information science, this article presents the results obtained from an analysis of these companies, comparing them with available data from previous studies. Types of services currently offered by these companies, their geographical distribution, business scope, size and age, current data on their employment and selection policies, and future prospects in the midst of the current economic crisis are described. Novel contributions of this report are the analysis of business scope and age of these companies and the factors they take into account when selecting their employees.
\end{abstract}

\section{Keywords}

Companies, Document services, Spain, Age, Size, Location, Employment, Recruitment, Future prospects. 
Paños-Álvarez, Antonio; Saura-Meroño, Pedro-José (2014). “Empresas españolas de gestión documental: servicios, organización, empleo y perspectivas". El profesional de la información, marzo-abril, v. 23, n. 2, pp. 144-150.

http://dx.doi.org/10.3145/epi.2014.mar.06

\section{Introducción}

El desarrollo de la sociedad de la información y del conocimiento y de las tecnologías de la información, la consideración de la información como recurso estratégico para empresas y organizaciones y la importancia de su gestión eficiente, ha generado nuevos modelos de negocio. A su vez ello ha traído como consecuencia la proliferación de empresas y profesionales de las tecnologías de la información y documentación dedicadas a ofrecer servicios en esas áreas de actividad.

En España, como en otros países, se han diversificado las empresas de productos y servicios documentales, que ofrecen gestión, asesoramiento, consultoría técnica y tecnológica, formación, gestión del conocimiento, etc.).

Este sector puede ofrecer empleos a titulados universitarios en información y documentación lo cual ha propiciado el interés de investigadores y profesionales en analizarlo. Citaremos algunos estudios publicados a partir del año 2000:

Se han estudiado entre otros los factores tecnológicos y normativos que han favorecido la expansión del sector en los últimos quince años (Baiget, 2006), el auge del perfil profesional y empresarial relacionado con la gestión documental y la gestión del conocimiento en las empresas y organizaciones (Bustelo-Ruesta; García-Morales, 2000; Cornella, 2000; Rodríguez-Rovira, 2000; Bustelo-Ruesta; García-Morales, 2001; Bustelo-Ruesta, 2003; Espinás, 2008).

Otros trabajos han estudiado el empleo en información y documentación en España, bien centrándose en el seguimiento de los demandantes de empleo, principalmente de los titulados universitarios (Tejada-Artigas; MoreiroGonzález, 2003; Borrego, Comalat; Estivill, 2004; MoreiroGonzález et al., 2008), bien en el estudio de las ofertas de empleo publicadas en prensa u otros medios (Alonso-Arévalo; Vázquez-Vázquez, 2000; Tejada-Artigas; RodríguezYunta, 2002), o bien mediante estudios que comparaban la situación con otros países como Francia (Tejada-Artigas; Rodríguez-Yunta, 2003) o Portugal (Frías; De-la-Mano; Moro-Cabero, 2008).

Algunos se centraron en el sector empresarial español, examinando sus tipos y los productos y servicios que ofrecen (Bustelo-Ruesta; García-Morales, 2000; Rodríguez-Yunta; Tejada-Artigas, 2003; 2004; Paños-Álvarez; Garrido-Alcolea, 2004). Otros posteriores, en fin, han estudiado las actividades empresariales que caracterizan el sector, la localización y datos de las empresas y cuestiones relativas a sus políticas de empleo (Tejada-Artigas; Rodríguez-Yunta, 2004; Rodríguez-Yunta; Tejada-Artigas, 2005).

Sin embargo, aunque el interés de estos estudios sigue teniendo importancia, en los últimos años han sido escasos. Por ello el objetivo de este trabajo es analizar cuál es la situación actual de este sector en el panorama español.

\section{Objetivos}

Partiendo de los datos suministrados en el Directorio de empresas españolas de servicios documentales elaborado en 2003 por el grupo Activa de Sedic, revisados en su edición de 2005 (Rodríguez-Yunta; Tejada-Artigas, 2005) y actualizados en este trabajo, el presente artículo pretende:

- clasificar el conjunto heterogéneo de empresas según los servicios ofertados;

- analizar su distribución geográfica, ámbito de negocio, antigüedad y tamaño;

- describir los perfiles formativos más demandados;

- estudiar las fuentes de reclutamiento y proceso de selección de nuevos empleados;

- analizar las expectativas de futuro de estas empresas.

\section{Metodología}

Después del escaso éxito de un primer intento de encuesta online, se realizó una encuesta telefónica con un cuestionario cerrado de 9 preguntas que permitió obtener los aspectos fijados como objetivos del presente trabajo. La población inicial (304 empresas) estaba formada por las empresas documentales del Directorio de Sedic (2005), que fue revisado y actualizado contactando con las empresas y a través de internet. 79 de ellas (un 26\%) ya no existían, lo que determinó una población de 225 empresas. De ellas, 124 (55\%) no quisieron contestar la encuesta aludiendo diversos motivos ${ }^{1}$. La muestra definitiva la formaron las 101 empresas que contestaron el cuestionario ${ }^{2}$. La encuesta se realizó durante los meses de junio y julio de 2012. Tras ello, se procedió al tratamiento de los datos, su tabulación y análisis con SPSS y la redacción de este trabajo.

\section{Análisis de los resultados}

\section{Clasificación de servicios documentales y tipos de empresas}

Se pidió a las empresas encuestadas que indicaran su actividad principal y hasta 2 actividades secundarias. A fin de hacer útiles estos datos, clasificamos dichas actividades conforme a la clasificación de servicios documentales propuesta en trabajos anteriores (Tejada-Artigas; RodríguezYunta, 2004; Rodríguez-Yunta; Tejada-Artigas, 2005) distinguiendo ahora 7 grupos de servicios ${ }^{3}$ (tabla 1 ).

Como puede observarse en la tabla 1, las empresas documentales españolas han experimentado cambios en sus actividades empresariales en los últimos 7 años. Así, el primer lugar en importancia lo ocupan ahora la gestión documental (43,6\% encuestadas lo señalan como su actividad principal y un $47,3 \%$ como secundaria). El diseño y distribución de software documental pasa a ocupar la segunda posición (el $29,7 \%$ lo marca como actividad principal, el $21,6 \%$ como secundaria y el $33,8 \%$ como tercera actividad). 
Tabla 1. Servicios documentales que ofrecen las empresas españolas analizadas en este trabajo $(n=101)$, identificando actividad principal, segunda ( $n=74)$ y tercera actividad $(n=71)$

\begin{tabular}{|c|c|c|c|}
\hline & Actividad principal (\%) & $2^{\mathrm{a}}$ actividad (\%) & $3^{\text {a }}$ actividad (\%) \\
\hline A. Consultoría en tareas de documentación & $13(12,9)$ & $11(14,9)$ & $11(15,5)$ \\
\hline B. Gestión documental & $44(43,6)$ & $35(47,3)$ & $28(39,4)$ \\
\hline C. Intermediación y distribución de contenidos & $9(8,9)$ & $4(5,4)$ & $2(2,8)$ \\
\hline D. Diseño web y edición electrónica de productos documentales & $3(3,0)$ & $3(4,1)$ & 0 \\
\hline E. Diseño y distribución de software documental & $30(29,7)$ & $16(21,6)$ & $24(33,8)$ \\
\hline F. Formación en archivística, biblioteconomía y documentación & $1(1,0)$ & 0 & $3(4,2)$ \\
\hline G. Otras actividades no relacionadas & $1(1,0)$ & $5(6,8)$ & $3(4,2)$ \\
\hline $\begin{array}{l}\text { Total empresas que han respondido indicando que ofrecen servicios de cada } \\
\text { tipo y por orden de importancia de dicha actividad }\end{array}$ & 101 & 74 & 71 \\
\hline
\end{tabular}

Número y (\% sobre el total de respuestas) de empresas que ofrecen cada tipo de servicios como actividad principal y como segunda o tercera actividad

Tabla 2. Servicios documentales que ofrecen las empresas españolas analizadas en este trabajo y comparación con los datos del estudio editado por Sedic (2005)

\begin{tabular}{|c|c|c|}
\hline & & \multirow[b]{2}{*}{ Sedic (2005) } \\
\hline & Paños-Álvarez; Saura-Meroño & \\
\hline A. Consultoría en tareas de documentación & $35(14,2)$ & $129(18,6)$ \\
\hline B. Gestión documental & $107(43,5)$ & $145(20,9)$ \\
\hline C. Intermediación y distribución de contenidos & $15(6,1)$ & $79(11,4)$ \\
\hline D. Diseño web y edición electrónica de productos documentales & $6(2,4)$ & $74(10,7)$ \\
\hline E. Diseño y distribución de software documental & $70(28,5)$ & $201(28,9)$ \\
\hline F. Formación en archivística, biblioteconomía y documentación & $4(1,6)$ & $67(9,6)$ \\
\hline G. Otras actividades no relacionadas & $9(3,7)$ & - \\
\hline $\begin{array}{l}\text { Total empresas que han respondido indicando que ofrecen servicios de cada tipo } \\
\text { (Cifras globales de nuestro estudio y del estudio de SEDIC 2005) }\end{array}$ & 246 & 695 \\
\hline
\end{tabular}

Número y (\% sobre total respuestas) de empresas que ofrecen actividades en cada tipo de servicios

Las cifras globales (tabla 2) de nuestro estudio y el de Sedic (2005) muestran ese cambio de tendencia, pues en 2005 el $20,9 \%$ de las empresas ofrecían servicios de gestión documental, mientras que ahora lo hace un $43,5 \%$ de las empresas encuestadas. Los servicios de diseño y distribución de software documental siguen manteniéndose, pues eran y son ofrecidos por alrededor del $28 \%$ de las empresas. Los servicios de formación han descendido su oferta (del 9,6\% en 2005 al 1,6\% de las empresas analizadas) y también los de intermediación y diseño web.

\section{Localización geográfica y ámbito de actividad}

La mayoría de las empresas documentales encuestadas se ubican en las comunidades de Madrid (41\%) y Catalunya (19\%), seguidas de Valencia, Andalucía y el País Vasco (gráfico 1), manteniéndose así las características de distribución señaladas en trabajos anteriores (Tejada-Artigas; Rodríguez-Yunta, 2004; Rodríguez-Yunta; Tejada-Artigas, 2005) ${ }^{4}$.

Como novedad respecto a estudios anteriores se preguntó a las empresas por su ámbito geográfico de actividad (tabla 3). A este respecto, hay que destacar que más de la mitad $(61,4 \%)$ orientan su negocio a España, mientras que un $38,6 \%$ también internacionalizan su actividad.

\section{Antigüedad y tamaño de las empresas}

Ningún estudio anterior examinó la antigüedad de las empresas documentales españolas. Los datos de nuestra en- cuesta señalan que el 5\% inició su actividad antes de 1980 y el $23 \%$ lo hizo a lo largo de la década de 1980 . Hay que destacar que en los 90 se produjo un fuerte avance de estas empresas, pues un $47 \%$ emprendió su negocio durante esos años y un $25 \%$ a partir del 2000 . Estos datos ponen de manifiesto la influencia que a partir de los 90 han tenido las tecnologías en el entorno bibliotecario y documentalista (Baiget, 2006). Con relación al tamaño y utilizando como criterio de clasificación la Recomendación 2003/303/EC de la Comisión Europea (atendiendo al número de empleados) (tabla 4), la distribución de las empresas es parecida a la del estudio de Sedic (2005), que indicaba que un $70 \%$ eran pequeñas y medianas empresas (pymes), pues se observa que el $75 \%$ de las analizadas ahora lo son.

Tabla 3. Ámbito geográfico de actividad de las empresas documentales analizadas $(n=101)$

\begin{tabular}{|c|c|c|c|}
\hline Autonómico & Regional & Nacional/Internacional & Nacional \\
\hline $5,9 \%$ & $6,9 \%$ & $38,6 \%$ & $48,6 \%$ \\
\hline
\end{tabular}

Tabla 4. Tamaño de las empresas analizadas $(n=101)$

\begin{tabular}{|l|l|}
\hline Pequeñas & $37 \%$ \\
\hline Medianas & $38 \%$ \\
\hline Grandes & $25 \%$ \\
\hline
\end{tabular}


Perfiles formativos más demandados

A diferencia de estudios anteriores, centrados en analizar el perfil de puestos de las empresas, hemos considerado interesante analizar las preferencias de perfil formativo que demandan estas organizaciones, más en línea con estadísticas de empleabilidad en entidades de demanda de empleo, que indirectamente informan sobre el perfil del puesto a desempeñar en la empresa, y cuyo análisis puede aportar valor añadido al curriculum formativo de los universitarios y de las titulaciones actuales. Así distinguimos en primera instancia el nivel de formación (grado o master) y en segundo lugar, el perfil de formación (documentación, informática u otras opciones).

Un $66 \%$ de las empresas analizadas manifestaron que el nivel de formación más demandado al contratar personal nuevo es el de graduado. Con relación al perfil, el 58\% indicó informática, el 39\% documentación, mientras que un 32\% indicó en porcentajes parecidos otros perfiles como: formación profesional administrativa, telecomunicaciones, economistas, periodistas, ingenieros o formación profesional.

\section{Fuentes de reclutamiento de nuevos empleados}

Las empresas analizadas muestran un estacionamiento en el uso del sistema público de empleo (Instituto Nacional de Empleo, INEM) y un mayor uso de internet en la búsqueda de nuevos candidatos, pues ya en $2004^{5}$, el $73,3 \%$ de las 30 empresas utilizaban la bolsa propia de trabajo, un 43,3\% convenios con universidades, un $40 \%$ las bolsas de internet y un $16,7 \%$ el INEM. En este análisis sobre 101 empresas, el $54 \%$ señaló el uso de webs de empleo y redes sociales, el $50 \%$ la propia web de la empresa, el $32 \%$ becas y convenios con universidades y un $16 \%$ el INEM.

\section{Modalidades de contratación}

La operativa sigue mostrando tendencias similares a las de años anteriores. Tejada-Artigas y Rodríguez-Yunta (2004) al analizar los resultados sobre una muestra de 28 empresas, indicaban una preferencia por los contratos por obra, segui-

Tabla 5. Requisitos más importantes en el proceso de selección de candidatos por orden de prioridad ( $n=101)$

\begin{tabular}{|c|c|c|c|c|c|c|}
\hline \multirow{2}{*}{$\begin{array}{c}\text { Orden de } \\
\text { prioridad }\end{array}$} & \multicolumn{5}{|c|}{ Requisitos } \\
\cline { 2 - 8 } & Experiencia & $\begin{array}{c}\text { Formación } \\
\text { universitaria }\end{array}$ & $\begin{array}{c}\text { Aptitud / } \\
\text { Discreción }\end{array}$ & Informática & Idiomas & Total \\
\hline $1^{\circ}$ & 57 & 25 & 8 & 7 & 3 & 101 \\
\hline $2^{\circ}$ & 12 & 28 & 22 & 28 & 11 & 101 \\
\hline $3^{\circ}$ & 4 & 3 & 36 & 35 & 23 & 101 \\
\hline
\end{tabular}

Número de empresas que priorizan cada factor como requisito en el proceso de selección de candidatos do por los contratos fijos y por becas. Los datos de las organizaciones encuestadas (101) indican que el $48 \%$ opta por los contratos temporales por obra o servicio, el $38 \%$ los fijos y un $14 \%$ los contratos de becarios y prácticas.

\section{Factores determinantes en el proceso de selección de candidatos}

A diferencia de trabajos anteriores, que manejan una amplia gama de factores de selección de personal, dada la metodología de recogida de datos que utilizamos (telefónica) que imponía rapidez en el trato con los encuestados, nos vimos obligados a reducir el número de factores a considerar, sintetizándolos en factores genéricos. Se pidió que indicaran por orden de prioridad cuáles eran los elementos más importantes a la hora de seleccionar a los candidatos en los procesos de selección de personal. Los resultados aparecen en la tabla 5.

La tendencia es seleccionar profesionales atendiendo en primer lugar a su experiencia (57,4\% de las empresas) y formación (24,8\%), para después analizar sus aptitudes y discreción, habilidades en informática y los idiomas.

Como segundo requisito, las empresas mostraron una paridad a la hora de señalar la formación universitaria y las habilidades informáticas $(27,7 \%)$, seguidas por las aptitudes y discreción de los candidatos en el desempeño de su trabajo $(21,8 \%)$. Los elementos de evaluación para el requisito 2o, están más alineados.

En el tercer requisito hay también cierta igualdad entre aptitud/discreción $(35,6 \%)$ e informática $(34,7 \%)$, apareciendo con cierto peso $(22,8 \%)$ en tercer lugar los idiomas. 
Hay tres variantes que destacan por encima de las demás: experiencia, informática y aptitud/discreción. Esto invita a reflexionar sobre la posible incorporación de contenidos prácticos (prácticas curriculares y extracurriculares) y tecnológicos (informática y análogos) en los actuales y futuros planes de estudios universitarios de grado y postgrado, que puedan mejorar las posibilidades de integración laboral de nuestros estudiantes.

La tendencia es seleccionar profesionales atendiendo en primer lugar a su experiencia y formación

\section{Tendencias de futuro}

Como novedad respecto a trabajos anteriores, y dada la situación actual de crisis, se consideró interesante preguntar a las empresas sobre la tendencia de su negocio en los próximos 5 años:

- 52\% señaló que se fijaba como objetivo de futuro un crecimiento que necesariamente pasaba por estrategias de diversificación de sus productos y servicios, apostando por la integración constante de las nuevas tecnologías, la formación de sus empleados y la automatización de sus servicios.

- $28 \%$ pensaba crecer siguiendo con su gama actual de productos y servicios.

- 17\% había apreciado visos de decrecimiento o estancamiento de sus negocios

- 3\% se planteaba estrategias de cambio de negocio, fusión con otras empresas, internacionalización de su actividad, y también el cierre.

\section{Conclusiones}

Este estudio muestra los aspectos que siguen igual y los que han cambiado en el panorama de las empresas españolas de servicios documentales en 2012, siete años después de los últimos estudios disponibles. Asimismo aporta información de valor del sector y algunas recomendaciones para la formación y el acceso al empleo.

La localización geográfica de las empresas analizadas muestra un comportamiento similar en 2012 al de 2005, pues se ubican de forma mayoritaria en las comunidades de Madrid y Cataluña, buscando quizás la proximidad a áreas de concentración de la administración y de las empresas a las que poder ofrecer sus productos y servicios. El tamaño también mantiene una distribución similar pues el $75 \%$ son pymes.

Sin embargo los servicios ofrecidos por las empresas han cambiado. En 2005 el 67\% de las 300 empresas incluidas en el directorio ofrecía servicios de diseño y distribución de software, el 48,3\% servicios de externalización de tareas documentales y un $43 \%$ consultoría en documentación, mientras que en la actualidad la muestra analizada (101) señala como primera actividad la externalización de servicios documentales $(43,6 \%)$ y en segundo lugar el diseño y distribución de software e informática $(29,7 \%)$, quedando en tercer lugar los servicios de consultoría en documentación $(12,9 \%)$. Sería interesante realizar estudios que analizaran las causas de este cambio de tendencia en las actividades de estas empresas.

Como aportaciones novedosas del estudio podemos señalar el análisis del ámbito de negocio y edad de estas empresas. El $48,6 \%$ se dedica al mercado nacional y algo más de un tercio, el $38,6 \%$, se orienta al mercado nacional/internacional. De estas últimas, el $87 \%$ se ubica en la comunidad de Madrid, el $34 \%$ en Cataluña y el $13 \%$ se distribuye entre País Vasco, Navarra y Valencia. Con relación a la antigüedad de las empresas cabe destacar que el $47 \%$ inició su actividad en la década de 1990, seguramente aprovechando la integración de las innovaciones tecnológicas en las empresas, y el $25 \%$ a partir de 2000.

Centrándonos en el ámbito laboral y de contratación de estas empresas podemos destacar varias cuestiones. Más de la mitad (66\%) prefieren contratar graduados a titulados con master. El perfil profesional y técnico más demandado es informático (58\%), seguido del titulado en documentación (32\%). Estos datos, junto con las actividades ofertadas por las empresas, pueden hacernos reflexionar sobre el valor que estas organizaciones dan hoy en día a la formación de posgrado y la necesaria combinación de formación en informática y documentación que deben buscar los titulados egresados en información y documentación, si quieren conseguir un puesto de trabajo.

A la hora de desarrollar el proceso de captación de empleados, las empresas analizadas siguen la tendencia que ya iniciaron en 2004 de disminución en el uso del servicio público de empleo. Actualmente, el $54 \%$ señala que prioriza el uso de webs de empleo y redes sociales, la mitad la propia web de la empresa, un tercio becas y convenios con las universidades y sólo un $16 \%$ el INEM. Esta información puede orientar a nuestros egresados universitarios sobre cuáles son los canales más eficientes a la hora de buscar empleo.

El perfil de contratación en las empresas analizadas sigue manteniéndose en términos similares a 2004, priorizando la contratación temporal (por obra o servicio), señalada por el $48 \%$ de las empresas.

El perfil profesional y técnico más demandado es informático $(58 \%$ de las empresas), seguido del titulado en documentación (32\%)

También como aportación singular de este trabajo, analizamos los factores más importantes para las empresas a la hora de seleccionar a sus candidatos. A este respecto, cabe destacar, que como requisito primero, más de la mitad $(57,4 \%)$ señalan la experiencia y el $24,8 \%$ la formación universitaria. Como requisito en segundo lugar, destacaron por igual la formación universitaria y las habilidades informáticas y en tercer lugar, también por igual destacaron las aptitudes y discreción de los candidatos y las habilidades informáticas. Dado que la experiencia sigue siendo el factor más cotizado, los egresados de información y documentación tendrán que aprender a sacar partido de las ofertas tem- 
porales de trabajo y/o prácticas que desarrollen, poniendo tales actividades en valor en su curriculum.

Otra aportación de este trabajo fue conocer la opinión de las empresas sobre su proyección de futuro (en los próximos 5 años), a fin de recabar información sobre el estado del sector. Las empresas son conscientes del difícil momento que viven, pues un $20 \%$ señala síntomas de estancamiento y/o decrecimiento, un $28 \%$ se fija como objetivo una política de crecimiento conservador (centrado en su gama actual de productos) y algo más de la mitad se plantea objetivos de crecimiento, que necesariamente pasarían por estrategias de diversificación de sus productos, apostando por la integración de las innovaciones tecnológicas y la mejora de la formación de sus trabajadores.

\section{Notas}

1. Los motivos alegados por la empresas fueron entre otros: "política de empresa; no contestan encuestas telefónicas; llame en otro momento; la persona responsable está reunida; no queremos contestar; no le puedo atender", y otras similares.

2. El error muestral para un nivel de confianza del $95 \%$ es un aceptable e $=0,072$. Con relación al sesgo de no respuesta y la validez externa de la muestra, cabe señalar que el t-test no determinó diferencias significativas entre las medias teórica y observada de las características de la muestra. Además se aprecia proporcionalidad con los estudios anteriores. Así, en torno al $70 \%$ de la población y la muestra son pymes, al igual que en el estudio de Sedic (2005).

3. Se distinguen los 6 grupos de actividades de los trabajos de Tejada-Artigas y Rodríguez-Yunta (2004) y Sedic (2005) y un grupo de actividades no relacionadas donde se integran las empresariales como la logística de mercancías y transportes, franquicias, gestión de obras de arte, almacenamiento y otras, que las empresas documentales han ido abordando como consecuencia de la crisis.

4. No se preguntó si las empresas tienen más de una sede u oficina por motivos de hacer más operativo el cuestionario, dado que se trataba de una encuesta telefónica y su extensión excesiva jugaba en contra de la tasa de respuesta. Se optó por dedicar las preguntas a obtener información sobre otras cuestiones interesantes como el ámbito de negocio.

5. Datos referenciados en el trabajo de Tejada-Artigas y Rodríguez-Yunta (2004) sobre una muestra de 30 empresas.

\section{Bibliografía}

Alonso-Arévalo, Julio; Vázquez-Vázquez, Marta (2000). "Características del comportamiento del mercado de trabajo en biblioteconomía, archivística y documentación. Bienio 98/99". Anales de documentación, n. 3, pp. 9-24. http://revistas.um.es/analesdoc/article/view/2531

Baiget, Tomàs (2006). “Les quinze coses que més han influït en la biblioteconomia i la documentació en els darrers quinze anys". Item, n. 43, pp. 69-90.

http://eprints.rclis.org/19497

Borrego, Andrés; Comalat, Maite; Estivill, Assumpció (2004). "Inserció laboral dels titulats en biblioteconomia i documentació per la Universitat de Barcelona”. Bid: textos universita- rios en biblioteconomía i documentació, junio, n. 12. http://bid.ub.edu/12borreg.htm

Bustelo-Ruesta, Carlota (2003). “Gestión documental y gestión de contenidos en las empresas: estado del arte 2002 y perspectivas para 2003". El profesional de la información, marzo-abril, v. 12, n. 2, pp. 118-120.

http://eprints.rclis.org/10039

Bustelo-Ruesta, Carlota; García-Morales, Elisa (2000). “La consultoría en organización de la información". El profesional de la información, septiembre, v. 9, n. 9, pp. 3-11.

http://www.elprofesionaldelainformacion.com/contenidos/2000/ septiembre/1.pdf

Bustelo-Ruesta, Carlota; García-Morales, Elisa (2001). "Tendencias en la gestión de la información, la documentación y el conocimiento en las organizaciones". El profesional de la información, diciembre, v. 10, n. 12, pp. 4-7.

http://eprints.rclis.org/10040

Cornella, Alfons (2000). "La gestión de la información en las organizaciones". El profesional de la información, abril, v. 9, n. 4, pp. 35-36.

Espinàs, Eulàlia (2008). “Una profesión en redefinición: visión desde el Col-legi Oficial de Bibliotecaris-Documentalistes de Catalunya". El profesional de la información, mayojunio, v. 17, n. 3, pp. 331-316.

http://www.elprofesionaldelainformacion.com/contenidos/2008/ mayo/07.pdf

http://dx.doi.org/10.3145/epi.2008.may.07

Frías, José-Antonio; De-la-Mano, Marta; Moro-Cabero, Manuela (2008). "La convergencia europea y la formación en Información y Documentación en la Universidad de Salamanca: proyectos y realidades". En: Frías, José-Antonio (Coord.): III Encuentro Ibérico de Docentes e Investigadores en Información y Documentación. Salamanca, pp. 217-224. ISBN: 9788474816549

Moreiro-González, José-Antonio; Azcárate, Pilar; Marzal, Miguel-Ángel; Tejada-Artigas, Carlos; Vergeiro, Waldomiro (2008). "Desarrollo profesional y opinión sobre la formación recibida de los titulados universitarios en información y documentación de las universidades públicas de Madrid (2000-2005)". El profesional de la información, mayo-junio, v. 17, n. 3, pp. 261-266.

http://www.elprofesionaldelainformacion.com/ contenidos/2008/mayo/02.pdf http://dx.doi.org/10.3145/epi.2008.may.02

Paños-Álvarez, Antonio; Garrido-Alcolea, Pedro (2004). "Estudio del modelo de productos y servicios de las empresas de servicios documentales". Anales de documentación, n. 7, pp. 199-213. http://eprints.rclis.org/12030/1/ad0713.pdf

Rodríguez-Rovira, Josep M. (2000). "Papel de las empresas consultoras en la gestión del conocimiento". El profesional de la información, septiembre, v. 9, n. 9, pp. 11-16. http://www.elprofesionaldelainformacion.com/contenidos/2000/ septiembre/2.pdf

Rodríguez-Yunta, Luis; Tejada-Artigas, Carlos (2004). "Recursos en internet sobre desarrollo profesional en documentación: 2. 
Empresas españolas de servicios documentales". Revista española de documentación científica, v. 27, n. 3, pp. 375-409.

http://redc.revistas.csic.es/index.php/redc/article/ viewFile/238/294

Rodríguez-Yunta, Luis; Tejada-Artigas, Carlos (2005). Servicios documentales: análisis y directorio de empresas españolas. Madrid: Sedic. ISBN: 8460951103

Tejada-Artigas, Carlos; Moreiro-González, José-Antonio (2003). “Mercado de trabajo en biblioteconomía y documentación. Estudios sobre la inserción laboral de los titulados universitarios". El profesional de la información, enerofebrero, v. 12, n. 1, pp. 4-9.

http://eprints.rclis.org/13045

Tejada-Artigas, Carlos; Rodríguez-Yunta, Luis (2002). Situa- ción laboral y desarrollo profesional de los socios de Sedic: resultados de la encuesta realizada en el 2001. Madrid. Sedic. ISBN: 8489456038

Tejada-Artigas, Carlos; Rodríguez-Yunta, Luis (2003). “De cara al espejo: imagen colectiva de los profesionales de la información en España y Francia". En: Los sistemas de información en las organizaciones: eficacia y transparencia. Fesabid 2003, VIII Jornadas Españolas de Documentación. Barcelona, pp. 431-442.

Tejada-Artigas, Carlos; Rodríguez-Yunta, Luis (2004). “Empresas españolas de servicios documentales: clasificación, tipología de servicios y encuesta sobre empleo". El profesional de la información, v. 13, n. 6, pp. 431-440.

http://eprints.rclis.org/1938

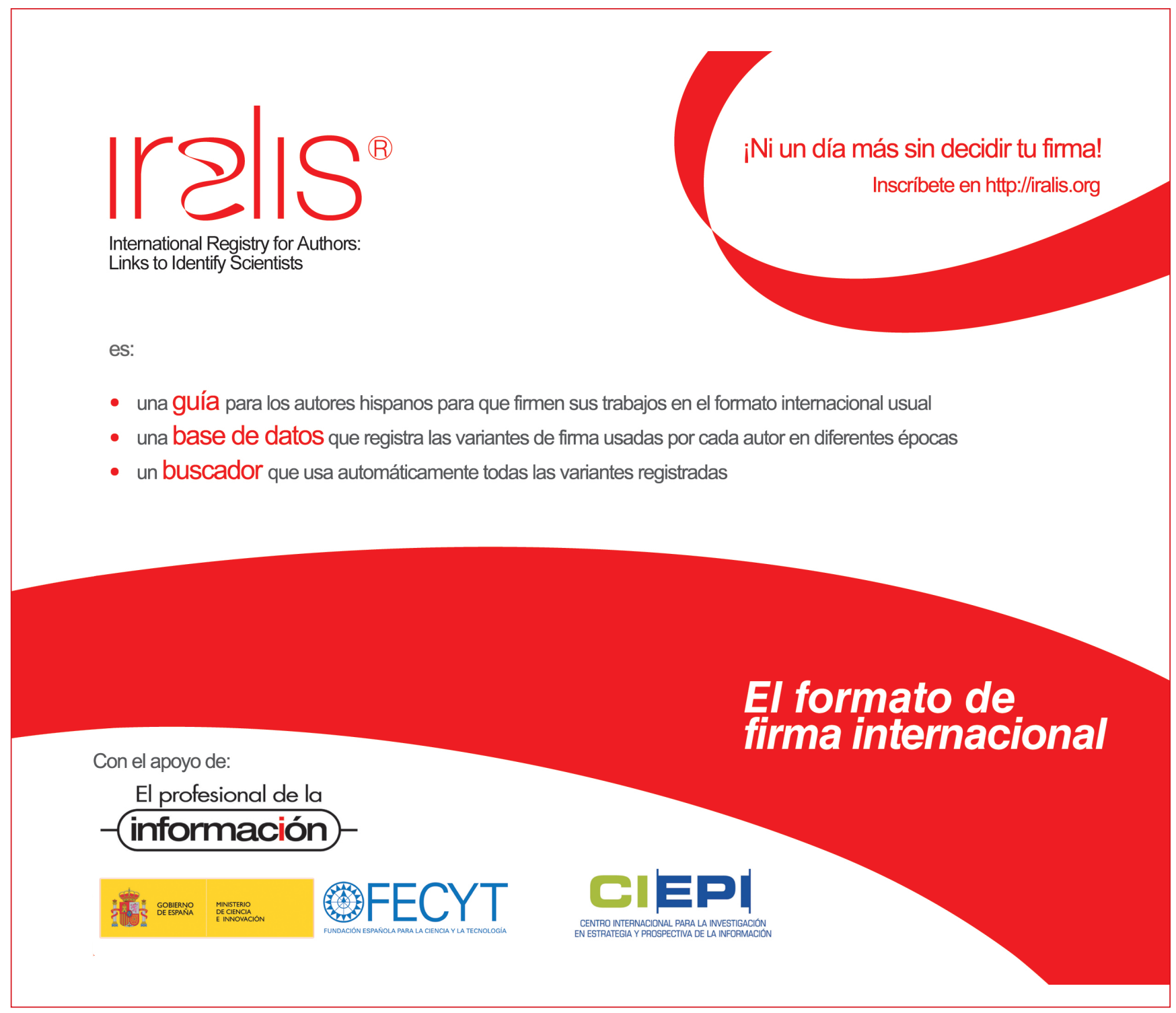

